\title{
Star-Shaped Metrics for Mechanical Metamaterial Design
}

\author{
JONÀS MARTÍNEZ, Université de Lorraine, CNRS, Inria, LORIA \\ MÉLINA SKOURAS, Université Grenoble Alpes, Inria, CNRS, Grenoble INP, LJK \\ CHRISTIAN SCHUMACHER, ETH Zürich \\ SAMUEL HORNUS, Université de Lorraine, CNRS, Inria, LORIA \\ SYLVAIN LEFEBVRE, Université de Lorraine, CNRS, Inria, LORIA \\ BERNHARD THOMASZEWSKI, Université de Montréal
}

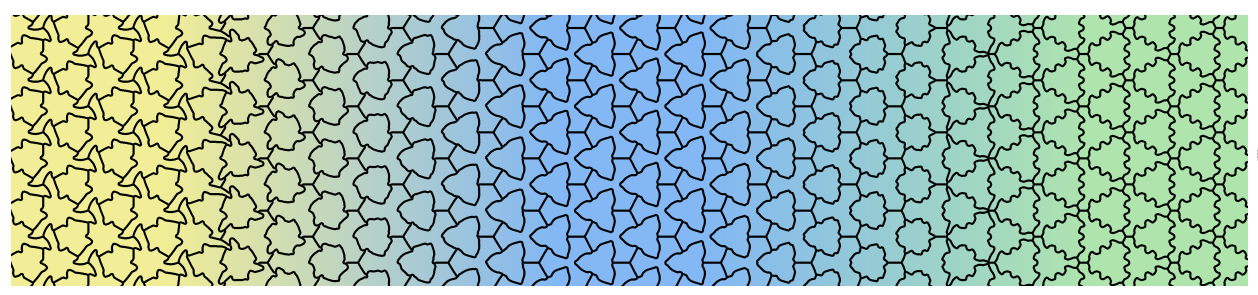

(a)
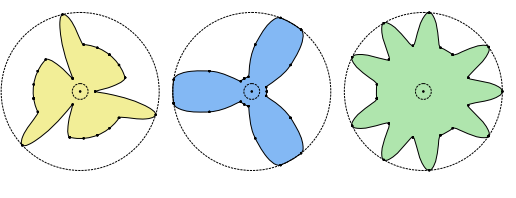

(b)

Fig. 1. Our method generates a smoothly-graded pattern (a) when interpolating between three star-shaped distance functions (b) on a regular honeycomb lattice. Each distance function is compactly parameterized with polar coordinates, allowing for simple interpolation in metric space as indicated by color-coding.

We present a method for designing mechanical metamaterials based on the novel concept of Voronoi diagrams induced by star-shaped metrics. As one of its central advantages, our approach supports interpolation between arbitrary metrics. This capability opens up a rich space of structures with interesting aesthetics and a wide range of mechanical properties, including isotropic, tetragonal, orthotropic, as well as smoothly graded materials. We evaluate our method by creating large sets of example structures, provided as accompanying material. We validate the mechanical properties predicted by simulation through tensile tests on a set of physical prototypes.

\section{CCS Concepts: • Computing methodologies $\rightarrow$ Shape modeling.}

Additional Key Words and Phrases: mechanical metamaterial, voronoi diagram, star-shaped metrics

\section{ACM Reference Format:}

Jonàs Martínez, Mélina Skouras, Christian Schumacher, Samuel Hornus, Sylvain Lefebvre, and Bernhard Thomaszewski. 2019. Star-Shaped Metrics for Mechanical Metamaterial Design. ACM Trans. Graph. 38, 4, Article 82 (July 2019), 13 pages. https://doi.org/10.1145/3306346.3322989

Authors' addresses: Jonàs Martínez, Université de Lorraine, CNRS, Inria, LORIA, F54000, Nancy, France; Mélina Skouras, Université Grenoble Alpes, Inria, CNRS, Grenoble INP, LJK, 38000, Grenoble, France ; Christian Schumacher, ETH Zürich; Samuel Hornus, Université de Lorraine, CNRS, Inria, LORIA, F-54000, Nancy, France; Sylvain Lefebvre, Université de Lorraine, CNRS, Inria, LORIA, F-54000, Nancy, France; Bernhard Thomaszewski, Université de Montréal.

Permission to make digital or hard copies of all or part of this work for personal or classroom use is granted without fee provided that copies are not made or distributed for profit or commercial advantage and that copies bear this notice and the full citation on the first page. Copyrights for components of this work owned by others than the author(s) must be honored. Abstracting with credit is permitted. To copy otherwise, or republish, to post on servers or to redistribute to lists, requires prior specific permission and/or a fee. Request permissions from permissions@acm.org.

(C) 2019 Copyright held by the owner/author(s). Publication rights licensed to ACM. 0730-0301/2019/7-ART82 $\$ 15.00$

https://doi.org/10.1145/3306346.3322989

\section{INTRODUCTION}

Digital manufacturing technologies such as 3D printing and laser cutting enable us to fabricate designs with great geometric detail. One particular way of exploiting this capability is to create patterned sheet materials whose geometric structures can be tailored to control their macro-mechanical behavior.

A typical approach to model and analyze structured sheet materials is centered around the concept of a representative element-a tile-which is repeated, transformed and laid out such as to generate a regular spatial tiling. Changing the shape of the representative tile allows for controlling macro-mechanical properties such as isotropy or negative Poisson's ratios. Generalizing this material design principle from a single representative tile to families of tiles that can be combined in a spatially-varying manner opens the door to structures with progressively-graded material properties.

There are several challenges that arise when defining such families of tiles. First, the space of tilings that can be generated from the tile family should span a wide range of macro-mechanical properties. At the same time, for applications such as fashion design or architecture, the shape of the tiles and the resulting patterns should also be aesthetically pleasing. Second, the tiles must satisfy a number of geometric constraints to ensure that they are tileable (boundary compatibility) and fabricable (minimum feature thickness). Lastly, the space of tiles should support progressive, robust gradation between different material properties.

In this work, we propose a new approach for generating tilings that simultaneously satisfy the above desiderata. Rather than producing a specific discrete set of tiles, our method defines a continuous space of tile geometries. Each point in this space corresponds to a tile shape that can cover the plane without gaps or overlaps. Furthermore, tile geometries can be robustly interpolated along any 
path within tile space. This interpolation property allows us to create spatially-varying tiles that combine into connected, smoothly graded structures. A macro-mechanical analysis of the space of tilings that can be generated with our approach reveals isotropic and orthotropic materials with a wide range of tensile stiffness and Poisson's ratio, including strongly auxetic structures.

Contributions. Our main contribution is to introduce a novel type of tiling for metamaterial design, allowing for robust interpolation and gradation of tile geometries. We obtain these tilings as Voronoi diagrams of regular lattices under star-shaped distance functions that generalize the Euclidean metric. A wide variety of geometries and macro-mechanical behaviors emerge when combining different metrics and lattice types. We study the connections between properties of the metric-in particular symmetries-and properties of the underlying lattice. This allows us to explore particularly relevant spaces of tilings that exhibit symmetries resulting in isotropic, tetragonal and orthotropic behaviors. In addition, the distance functions can be varied across the underlying lattice, producing heterogeneous graded networks. Finally, we show a wide range of possible tilings combining diverse mechanical behavior with different aesthetics. We fabricate a number of networks to illustrate potential applications in mechanical engineering and design.

\section{RELATED WORK}

Structural Patterns. Tilings and patterns, intriguing from a mathematical point of view [Grünbaum and Shephard 1986], have long been used for their particular aesthetics, e.g. for wall and floor coverings, stained-glass windows, in art (see e.g. the Escher-inspired tesselations by Kaplan and Salesin [2000]) or in architecture (see e.g. the roof of the Louvre Abu Dhabi or the façades of the MuCEM in Marseille). When tilings are used as structural elements, as is often the case in architectural and engineering applications, or when the objects are meant to be manipulated, not only the visual properties but also the mechanics need to be accounted for when designing the tiles. With these considerations in mind, Dumas et al. [2015] recently proposed an algorithm to simultaneously optimize the appearance and the structural soundness of on-surface uniform textures based on user-provided pattern exemplars. In order to address specific loading scenarii, Martínez et al. [2015] combined topology optimization and by-example texture synthesis in a single optimization problem. A related approach, also based on topology optimization and combined objectives, was later presented by Schumacher et al. [2016] to cut out decorative patterns from three-dimensional surfaces while preserving their stability. Focusing on filigrees, i.e. thin patterns composed of repeated base elements, Chen et al. [2016] proposed an automatic packing technique ensuring that individual base elements are well connected and satisfy fabrication constraints. Zehnder et al. [2016] proposed an interactive tool that lets the user control the placement of the elements on the target surface and guides them in strengthening the final structure.

Metamaterials. Modifying the inner structure of a tile or its threedimensional counterpart will affect its mechanical behavior, which can be leveraged to engineer new materials, so-called metamaterials. The macro-mechanical properties of the resulting tiling can be obtained by fitting a homogeneous material model, a technique called homogenization [Hassani and Hinton 1998]. As shown by Schumacher et al. [2018], this approach is not limited to linear materials and can be adapted to characterize the nonlinear behavior of structured sheet materials, similar to the ones that we generate in this work. Metamaterials typically exhibit a much wider range of properties than their base materials. For example, they may be anisotropic when their base materials are not or offer improved trade-offs between density and stiffness. Metamaterials can even have properties that are not commonly found in nature, as in the case of auxetic metamaterials, i.e. materials with a negative Poisson's ratio that will expand transversally when stretched along a give direction [Babaee et al. 2013; Kolken and Zadpoor 2017; Lakes 1987; Liu and Zhang 2018]. As such, they are of great interest to engineers and material scientists and actively studied in these communities. Auxetic mechanical metamaterials can be obtained, for instance, by an arrangement involving rigid squares connected together at their vertices by hinges [Grima and Evans 2000], or by performing specific star-shaped perforations [Mizzi et al. 2018]. We refer to Bertoldi et al. [2017] for a recent overview. While many metamaterials were originally designed by hand, computational approaches relying on, e.g., topology optimization, proved very useful to automatically generate metamaterials with prescribed properties [Andreassen et al. 2014; Sigmund 1997; Wang et al. 2014].

Heterogeneous Designs. Metamaterials can be directly used for designing objects with homogeneous bulk properties. In practice, however, many applications involve objects with spatially varying materials. These materials are either manually selected by the designer or result from an inverse modeling procedure that optimizes the materials of the object such that it behaves as desired [Bickel et al. 2010; Xu et al. 2015]. While compatible building blocks can be combined to propagate mechanical signals in certain contexts [Coulais et al. 2016; Ion et al. 2016, 2018], using metamaterials for heterogeneous designs, in the general case, is particularly challenging since one needs to ensure that only materials corresponding to fabricable microstructures are used and that adjacent microstructures are properly connected. Leveraging the fact that metamaterials with different topologies may exhibit similar macro material properties, Zhu et al.[2017] solve large-scale topology optimization problems by directly working with continuous properties and greedily fixing interface discrepancies between adjacent tiles when mapping back materials to microstructures. Garner et al. [2019] simultaneously optimize for physical properties and connectivity by including a term in the optimization objective that favors compatible microstructures. Schumacher et al. [2015], employ a message-passing based algorithm to synthesize microstructures with matching boundaries. However, these techniques do not guarantee that no mismatch between adjacent tiles occurs, and they are not well adapted to metamaterials made of thin patterns. To avoid interface discrepancies, another approach is to introduce explicit connectivity constraints during topology optimization [Du et al. 2018; Zhang et al. 2018; Zhou and Li 2008]. A popular alternative is to rely on parametric families of microstructures sharing the same topology at the boundaries. For example, Panetta et al.[2015] generate tileable families of truss-like 
structures that can be further optimized to avoid stress concentration [Panetta et al. 2017]. Schumacher et al. [2015], Cramer et al. [2016] and Wang et al. [2017; 2018] rely on signed-distance functions to represent and grade microstructures with similar topology. Konaković et al. [2016] and Malomo et al. [2018] exploit parametric auxetic metamaterials to optimize two-dimensional plates that can assume three-dimensional custom shapes when deformed. In a similar vein, Konaković-Luković [2018] investigate the design of spatially-graded auxetic metamaterials that can be deployed into desired shapes. However, restricting materials for the inverse problems to originate from a single family of microstructures, selected a priori, limits the space of achievable behaviors and therefore the scope of the proposed methods. .

Building on the work of Martínez et al. [2018], who define microstructures as Voronoi diagrams based on convex polyhedral distances, we propose to use star-shaped distance functions to generate periodic metamaterials. As opposed to the convex setting of Martínez et al. [2018], star-shaped metrics lead to Voronoi cells which are not necessarily star-shaped sets, thus spanning a wider range of achievable Voronoi cell geometries. Instead of grading material properties by interpolating microstructures directly as commonly done, we interpolate the star-shaped metrics. The graded microstructure then follows as the Voronoi diagram induced by these spatially-varying metrics. This approach allows us to achieve proper connectivity between any pair of metamaterials of our databases. Thus, our metamaterials are as easy to grade as aperiodic microstructures, such as the isotropic and orthotropic foams of Martínez et al [2016; 2017], while covering a wider material space and exhibiting more diverse aesthetics.

\section{METHOD}

The basic idea of our work is to design mechanical metamaterials through generalized Voronoi diagrams induced by star-shaped metrics. We start by introducing the concept of star-shaped metrics and show some of their basic properties (Section 3.1). We then characterize their induced Voronoi diagrams (Section 3.2) and describe an algorithmic implementation of star-shaped Voronoi diagrams that, by construction, guarantees cell connectedness (Section 3.3). We generalize our formulation to spatially-varying metrics in Section 3.4. Finally, we study the relation between distance and lattice symmetries and how they translate into symmetries of the Voronoi diagram (Section 3.5).

\subsection{Star-shaped Metrics}

Definition. Let $\mathcal{S} \subset \mathbb{R}^{2}$ be a compact set containing the origin $O$ in its interior. We call $\mathcal{S}$ star-shaped with respect to $O$ if for any point $x \in \mathcal{S}$, the line segment $[x, O]$ is contained in $\mathcal{S}$. The star-shaped distance induced by $\mathcal{S}$ from a point $p$ to a point $q$ is

$$
d_{\mathcal{S}}(p, q)=\min \{t \geq 0: q \in p+t \mathcal{S}\},
$$

i.e., $d_{\mathcal{S}}(p, q)$ is the smallest factor by which we have to scale $\mathcal{S}$, centered around $p$, such that $q$ lies on its boundary.

Since $\mathcal{S}$ is an star-shaped set its boundary can be parameterized in polar coordinates by a continuous and periodic function $f: \mathbb{R} \mapsto$ $[a, b]$, where $0<a \leq b$ are the minimum and maximum radial span of the distance (see Figure 2a). By doing so, the distance $d_{\mathcal{S}}(p, q)$ is equivalent to the formulation of Icking et al. [2001],

$$
d_{\mathcal{S}}(p, q)=\frac{\|q-p\|}{f(\angle(q-p))},
$$

where $\angle(\cdot)$ is the angle of a vector with respect to the horizontal axis, and $\|\cdot\|$ is the Euclidean norm (see Figure $2 b$ ). Note that

$$
\frac{\|q-p\|}{b} \leq d_{\mathcal{S}}(p, q) \leq \frac{\|q-p\|}{a} .
$$

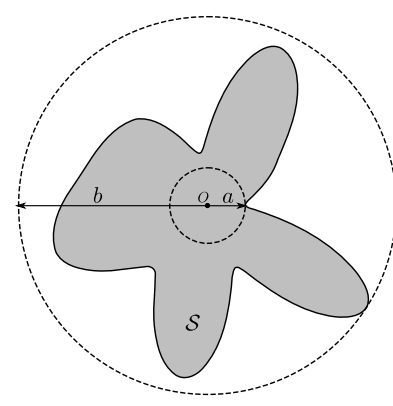

(a)

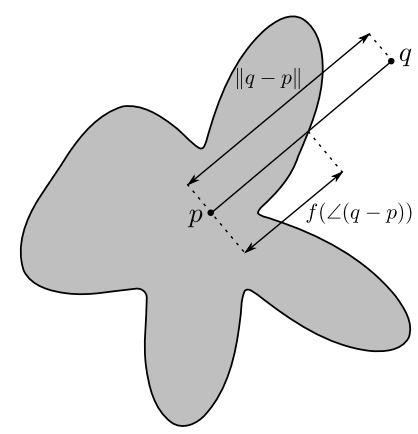

(b)
Fig. 2. Illustration of star-shaped distance. 2a: a star-shaped set $\mathcal{S}$ whose radial span is bounded by $a$ and $b$. 2b: the distance from point $p$ to $q$ as defined by Equation 2.

Properties. Similar to the case where $\mathcal{S}$ is a convex set [Icking et al. 2001], the following properties hold:

$$
\begin{gathered}
d_{\mathcal{S}}(p, q) \geq 0 \quad \text { (non-negativity) } \\
d_{\mathcal{S}}(p, q)=0 \Leftrightarrow q=p \quad \text { (definiteness) } \\
\forall \lambda \geq 0, d_{\mathcal{S}}(\lambda p, \lambda q)=\lambda_{\mathcal{S}}(p, q) \quad \text { (positive homogeneity) } \\
d_{\mathcal{S}}(p, q)=d_{\mathcal{S}}(q, p) \Leftrightarrow \mathcal{S} \text { is a centrally symmetric set }
\end{gathered}
$$

However, contrary to the case of convex distances, the triangle inequality does not necessarily hold.

Parameterization. The space of star-shaped distances is vast, and we seek to parameterize it with few parameters. We encode distances at equally spaced angles and interpolate in-between. Consider an increasing sequence of $n \geq 3$ angles $\left(\alpha_{1}, \ldots, \alpha_{n}\right)$, with $\alpha_{n}-\alpha_{1}<2 \pi$, and their corresponding radial spans $\left(l_{i}=f\left(\alpha_{i}\right)\right)_{i}$. We consider two different interpolations for $f$ :

- Polygonal outline. Interpolation of $f$ given by a simple polygon with vertices $\left[l_{i} \cos \left(\alpha_{i}\right), l_{i} \sin \left(\alpha_{i}\right)\right]$ (see Figure 3a). In that case, we respect the additional constraint $\forall i, \alpha_{i+1}-\alpha_{i}<\pi$.

- Smooth outline. $f$ is interpolated (in polar coordinates) with periodic cubic interpolation. In order to satisfy the bounds in Equation 3, we use the monotonic interpolation method of Fritsch and Carlson [1980] (see Figure 3b).

Other types of interpolation are possible as well, e.g., based on circular harmonics.

\subsection{Voronoi Diagrams induced by Star-shaped Metrics}

In this section, we define the Voronoi diagram induced by starshaped distance functions and study some of their properties. 


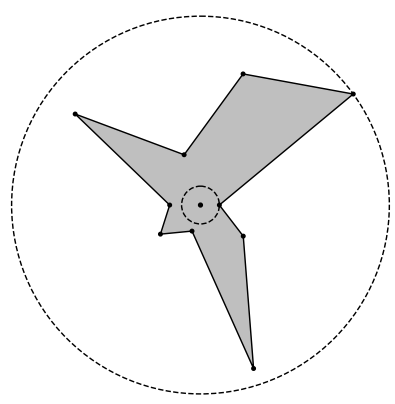

(a) Polygonal

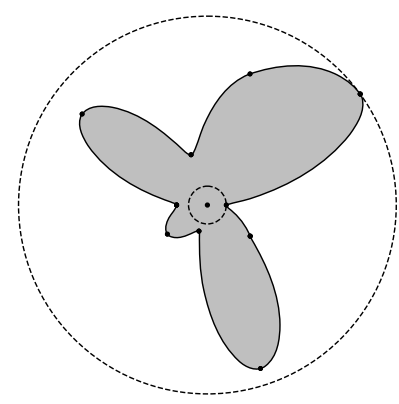

(b) Smooth
Fig. 3. Two different interpolations of 10 equidistant angular samples and their corresponding radial span.

Definition. Let $\Gamma$ be a set of sites in $\mathbb{R}^{2}$. The Voronoi diagram over $\Gamma$ induced by the distance $d_{\mathcal{S}}$ is the decomposition of $\mathbb{R}^{2}$ into Voronoi cells

$$
V_{\mathcal{S}}(p)=\left\{x \in \mathbb{R}^{2}: \forall q \in \Gamma \backslash p, d_{\mathcal{S}}(p, x)<d_{\mathcal{S}}(q, x)\right\},
$$

where $p \in \Gamma$. The bisector between two sites $p$ and $q$ is

$$
\mathcal{B}(p, q)=\left\{x \in \mathbb{R}^{2}: d_{\mathcal{S}}(p, x)=d_{\mathcal{S}}(q, x)\right\}
$$

Properties. We now discuss an important property which leads to an efficient, robust algorithm to extract the Voronoi cells of a set of sites under a star-shaped distance function. In short, given an assumption on the set of sites, the extent of the Voronoi cell of a site is bounded as long as the ratio between the maximum and minimum radius of the star-shaped distance is bounded.

LEMMA 3.1 (BOUNDED SIZE OF VORONOI CELLS). Let $\Gamma$ be a set of sites such that $\forall y \in \mathbb{R}^{2}, \exists q \in \Gamma$ such that $\|y-q\| \leq k$; that is, for any point in the plane there is a site of $\Gamma$ at a (Euclidean) distance less than $k$ away. Let $p$ be a site in $\Gamma$. Then, $\forall x \in V_{\mathcal{S}}(p)$ we have that $\|x-p\| \leq k \frac{b}{a}$.

Proof. Let $x$ be a point in $V_{\mathcal{S}}(p)$. By contradiction, let us assume (i): $\|x-p\|>k \frac{b}{a} \geq k$. By assumption on our set of sites, there exists a site $q$ such that $\|x-q\| \leq k$. By $(i), q$ is different from $p$. We thus have

$$
d_{\mathcal{S}}(q, x) \leq \frac{\|q-x\|}{a} \leq \frac{k}{a}<\frac{\|p-x\|}{b} \leq d_{\mathcal{S}}(p, x),
$$

which is a contradiction since $x \in V_{\mathcal{S}}(p)$.

Lemma 3.1 implies that for $k<\infty$, the maximum achievable radius of Voronoi cells increases proportionally to the ratio $\frac{b}{a}$. Examples of point sites with $k<\infty$ include periodic lattices (see Figure 15), which is our focus in this work. This lemma will be used in the algorithm presented in Section 3.3 to establish a proper finite extent of computation.

Voronoi diagrams and bisectors of sites under general convex distances have been extensively studied [Blömer and Kohn 2018; Corbalan et al. 1996; Deza and Sikirić 2015; He et al. 2011; Icking et al. 2001; Ma 2000; Martini and Swanepoel 2004; Väisälä 2013]. The interested reader can find a global classification of distances for Voronoi diagrams in chapter 20 of the textbook by Deza and Deza [2013]. To the best of our knowledge, the only works considering the Voronoi diagram of star-shaped distances are a series of three papers by Gruber [1974], where it was shown that bisectors are fully contained in a single hyperplane if and only if the distance is ellipsoidal and strictly convex.

The bisector structure of two sites $\mathcal{B}(p, q)$ is characterized by the boundary set $\partial \mathcal{S}$ [Ma 2000]. This is convenient since it allows to determine the linearity of bisectors. In particular, for polygonal distance outlines, it is known that the bisector is composed of straight line segments [Ma 2000]. For smooth distance outlines, $\mathcal{B}(p, q)$ is composed of curves. This is illustrated in Figure 4.

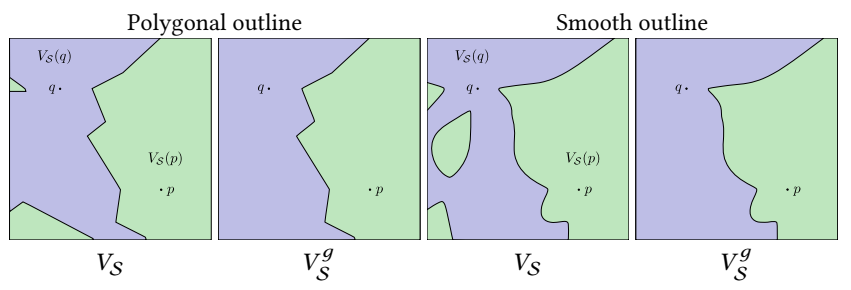

Fig. 4. Voronoi cells (distance model $V_{\mathcal{S}}$ and Voronoi growth model $V_{\mathcal{S}}^{g}$ ) for two points $p$ (in green) and $q$ (in purple), considering the two different distance outlines shown in Figure 3.

Compared to their Euclidean counterparts, Voronoi diagrams induced by star-shaped distances have unusual properties. In particular, Voronoi cells are not necessarily connected sets. For the case of convex distances, it is known that the bisector is homeomorphic to a line and that Voronoi cells are star-shaped sets [Corbalan et al. 1996; Icking et al. 1995]. However, for star-shaped distances the bisector $\mathcal{B}(p, q)$ is not in general homeomorphic to a line (see Figure 5). This implies that a Voronoi cell may have more than one connected component. This is a potentially severe issue for fabrication as disconnected curves will exist in the geometry.

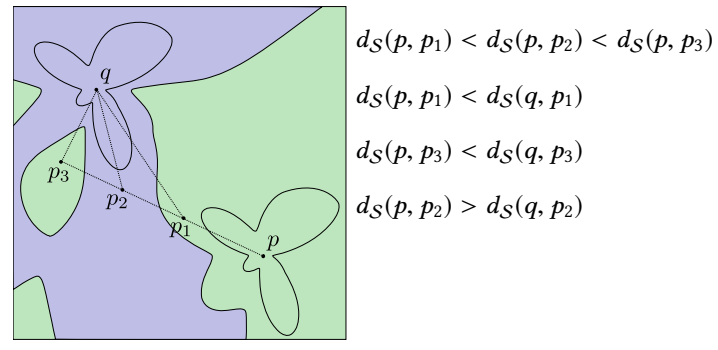

Fig. 5. Star-shaped metrics can lead to cells with non-trivial topology. In this example, the strongly non-convex, anisotropic metric leads to the formation of a green island deep in the purple territory of site $q$. This phenomenon is explained by the inequalities on the right.

\subsection{Growing Voronoi Diagrams with Star-shaped metrics}

To ensure that cells are connected sets, we adopt the Voronoi growth model [Avrami 1939; Zayer et al. 2018]. In this model, the Voronoi cells are defined through an isotropic growth process based on $\mathcal{S}$. 
Visually, the cells for all sites are grown simultaneously in an initially isotropic manner, synchronized through a global time parameter. The resulting plane partition is similar to what one would obtain by iteratively replacing each connected component $K$ of $V_{\mathcal{S}}(p)$ that does not contain its site $p$, by the restriction to $K$ of the Voronoi diagram of the sites whose cell surround $K$. For efficiency, however, the algorithm uses front-propagation (see below). The growth-based Voronoi cell of site $p$ is denoted $V_{\mathcal{S}}^{g}(p)$. By construction, the cell $V_{\mathcal{S}}^{g}(p)$ is always a connected set, as illustrated in Figure 4.

In the following, we use the term Voronoi network to refer to the set difference between $\mathbb{R}^{2}$ and the Voronoi cells, i.e., $\mathbb{R}^{2} \backslash\left(\bigcup_{p \in \Gamma} V_{\mathcal{S}}^{g}(p)\right)$ The geometry of the Voronoi network is both influenced by the distance and the specific distribution of the set of sites $\Gamma$. We are particularly interested in the case where the set of sites is a lattice. Note that the same metric may produce different Voronoi networks depending on the type of lattice (see Figure 6).

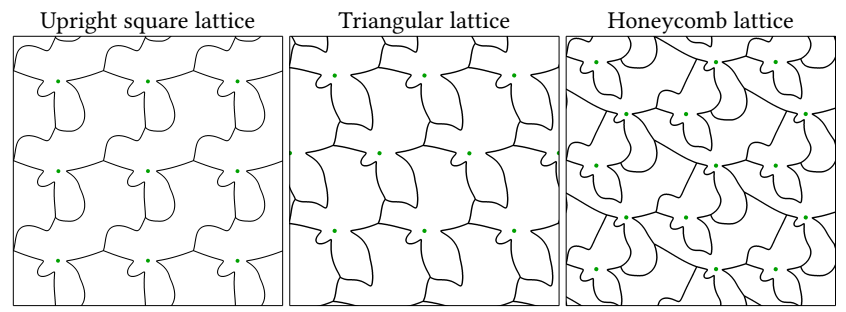

Fig. 6. Voronoi networks generated with the Voronoi growth model (distance Figure 3b, smooth outline) using three different types of lattice (green points).

It is known that convex distance functions lead to Voronoi cells that are star-shaped sets with respect to their sites [Ma 2000]. Fortunately, general star-shaped distances cover a wider space of achievable Voronoi cell geometries, as illustrated in Figure 6.

Algorithm. We compute the discrete Voronoi cells of $\Gamma$ with a floodfill algorithm that emulates the Voronoi growth process in a finite extent. The algorithm outputs a two-dimensional Cartesian grid $G$ whose cells are labeled according to the Voronoi cell to which they belong (see pseudocode in Algorithm 1). The algorithm operates on a priority queue which holds pairs $(p, c)$ of grid cells $c \in G$ (the center of the grid cell) and sites $p \in \Gamma$, ordered according to their distance $d_{\mathcal{S}}(p, c)$. Whenever an element of the queue is processed, we check whether its grid cell is still unlabeled and, if so, label it according to the site of the current element. We then add elements for the four neighbors of the cell and repeat until the queue is empty. Figure 7 shows the progression of the algorithm for two sites.

When computing the Voronoi cells of periodic lattices, we extend the domain of computation and return the result in the unextended domain. More precisely, we extend the domain according to the upper bound on the size of Voronoi cells given in Lemma 3.1, that strictly holds for the Voronoi growth model. For instance, for $a=0.2$, $b=1$, and $k=1$, we expand the domain by $k \frac{b}{a}=5$.

The Voronoi growth model allows us to add morphological constraints on the geometry of the Voronoi cells and, consequently, on the resulting Voronoi network. By simply discarding tuples $(p, c)$ in
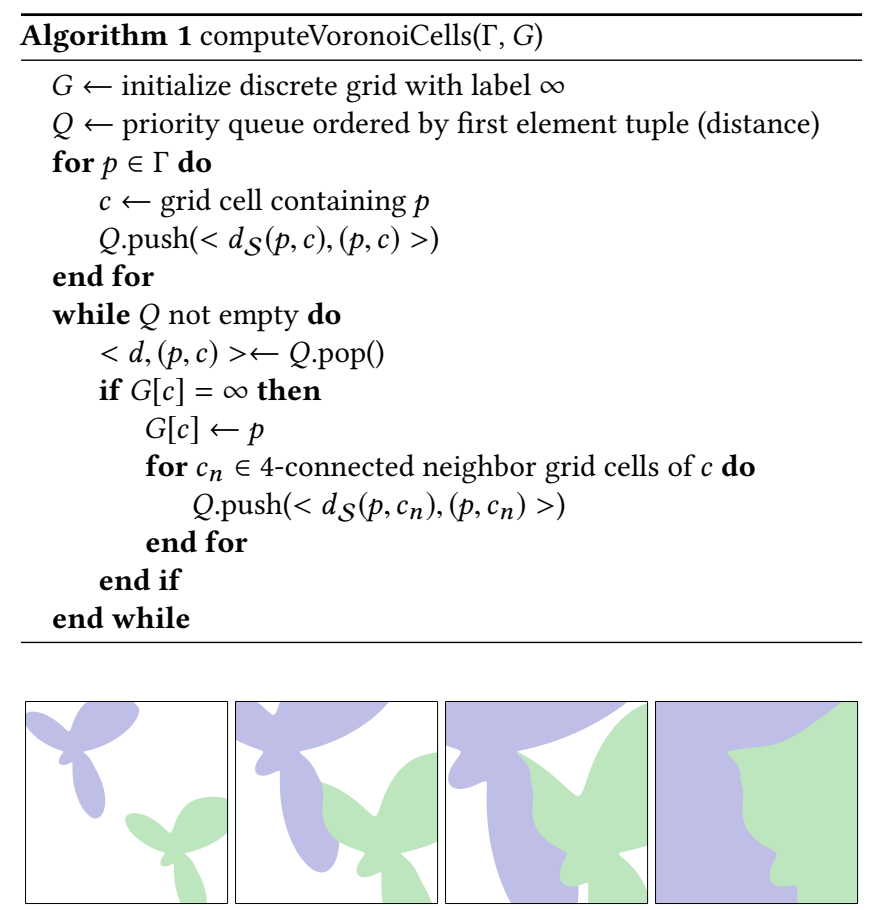

Fig. 7. From left to right: evolution of the flood-fill algorithm applied to the smooth case shown in Figure 4 using a grid with $1000^{2}$ cells.

Algorithm 1 whose discrete Euclidean neighborhood of radius $r>0$ (relative to the cell size of $G$ ) around $c$ contains at least one grid cell $c^{\prime}$ such that $G\left[c^{\prime}\right] \neq \infty \wedge G\left[c^{\prime}\right] \neq p$, we can ensure that the Voronoi network corresponds to the Minkowski sum given by a discrete disk of radius $r$. This is particularly well-suited for fabrication since it provides a simple way of enforcing minimum-thickness constraints on the Voronoi network. Figure 8 provides an illustration.

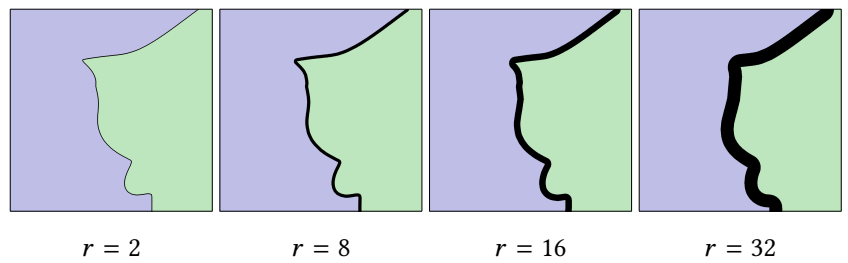

Fig. 8. Restricted Voronoi growth for the smooth case shown in Figure 4 with different neighborhood radii $r$, as indicated, on a grid with $1000^{2}$ cells.

The discrete flood fill algorithm is simple to implement and robust. However, some degenerate cases may arise. For instance, it is challenging to deal with distances coinciding at exactly the same point with equal growth rate. Fortunately, considering a maximum radius of growth $r$ or increasing the resolution of $G$ resolves such degenerate configurations in most (but not all) cases; see Figures 9 and 20. Alternatively, one may resort to more advanced techniques to cope with degenerate cases [Edelsbrunner and Mücke 1990]. 


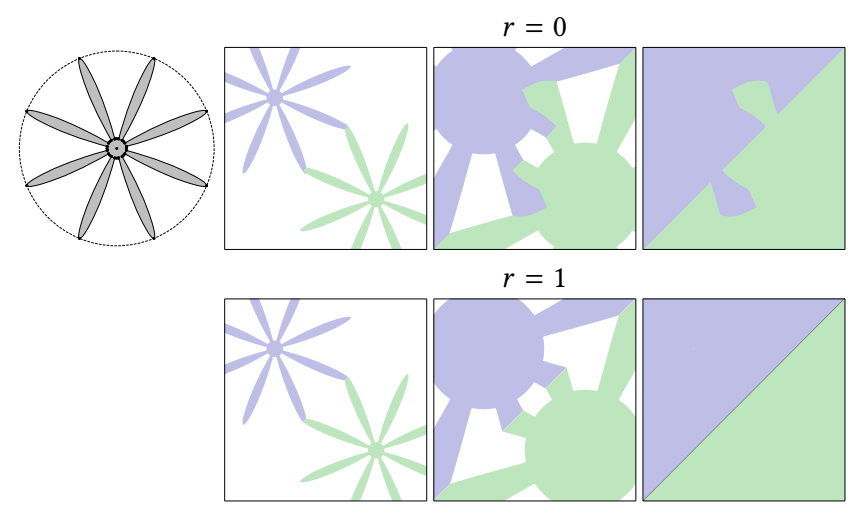

Fig. 9. A centrally symmetric smooth distance (top left) with eight equal petals meeting at four different points during the growth (on a grid with $1000^{2}$ cells). Without restricting the growth $(r=0)$, one of the fronts randomly advances through the other, due to the finite resolution of the grid cell. In this case, restricting the growth $(r=1)$ stabilizes the two fronts, effectively eliminating the degeneracy.

\subsection{Spatially Varying Star-shaped Distances}

In the examples shown so far, the metric was constant throughout the domain. However, our formulation generalizes without modification to spatially-varying metrics. We use this capability to design metamaterials with spatially-grade mechanical properties (see Section 4.3). Before we proceed to analyze the mechanical properties of the structures they induce, we will introduce two ways of generating spatially-varying metrics.

Discrete Metric Assignment. The simplest option is to assign different metrics to different sites, according to some assignment function $M: \Gamma \rightarrow \mathcal{S}$, and to reconstruct the Voronoi diagram as before. This approach allows us to implement hard-but-continuous transitions between two structures as shown in Figure 10, left. It is also possible to generate local metric variations, such as the red-black assignment of two different metrics in Figure 10, right.

Continuous Metric Interpolation. The second option is to define a smoothly-varying metric field by means of interpolation. While more complex schemes are possible, we restrict considerations to simple linear interpolation. In the continuous case, this amounts to interpolating between two star-shaped sets,

$$
\mathcal{S}(\beta)=\mathcal{S}_{1} \beta+\mathcal{S}_{2}(1-\beta), \quad \beta \in[0,1],
$$

and constructing the distance function on the interpolated set as defined in (1). Figures 10 and 11, show examples of networks obtained by linearly interpolating two given metrics in different directions; we defer a detailed analysis of the properties of these graded structures to Section 4.2 .

\subsection{Distance and lattice symmetries}

Due to the complex relationship between metric shape, lattice, and induced Voronoi diagram, predicting the resulting tile geometries is difficult. Nevertheless, some geometric properties of the metrics and lattices are reflected in the resulting Voronoi diagrams. In particular, symmetries of the metric and the lattice translate into tiling symmetries, which in turn induce material symmetries. As we will see in Section 4.1, this property can be used to design materials which are isotropic or orthotropic by construction.

We focus our analysis of symmetries on the growth-based Voronoi diagrams induced by $n$-fold rotationally-symmetric and $n$-reflection symmetric star-shaped metrics (see Figure 12). Table 1 summarizes the symmetry relations that arise on triangular, square, and honeycomb lattices.

Periodicity. The lattices that we consider are periodic and can be obtained by translating a primitive cell, in our case a regular polygon, along two or three directions. By construction, our growing - and therefore grown - Voronoi cells are also invariant under the same translations, and so must be the resulting bisectors. Consequently, the Voronoi diagrams that we obtain are also periodic.

Rotational symmetry. Let $R_{\theta}$ denote the rotation around $O$ by an angle $\theta$ and $\mathcal{S}$ a compact set invariant under $R_{\theta}$, i.e. a set for which $R_{\theta} \mathcal{S}=\mathcal{S}$. Then rotating a translated set $\mathcal{S}_{p}=p+\mathcal{S}$ by $\theta$ is equivalent to translating the set $\mathcal{S}$ to the rotated point $R_{\theta} p$, since $R_{\theta}(p+\mathcal{S})=R_{\theta} p+R_{\theta} \mathcal{S}=R_{\theta} p+\mathcal{S}$ (see Figure 13, top left).

Let $x_{i}, 0 \leq i \leq n-1$, denote the vertices of a regular $n$-sided polygon in counterclockwise order such that $x_{i}=R_{\frac{i 2 \pi}{n}} x_{0}$. According to the above property, if $\mathcal{S}$ is $n$-fold rotationally-symmetric, the fan of translated sets $\mathcal{S}_{i}=x_{i}+\mathcal{S}$ are rotated copies of $\mathcal{S}_{0}$, as $R_{\frac{i 2 \pi}{n}} \mathcal{S}_{0}=R_{\frac{i 2 \pi}{n}} x_{0}+\mathcal{S}=x_{i}+\mathcal{S}=\mathcal{S}_{i}$ (Figure 13, bottom left). Consequently, this polygonal configuration of sets is $n$-fold symmetric. This implies, in particular, that a square, or diagonal square, arrangement of four copies of a four-fold rotational symmetric compact set is four-fold symmetric and that a hexagonal arrangement of six copies of a six-fold rotationally-symmetric compact set is six-fold symmetric. Therefore, since a square lattice is also four-fold symmetric itself, a periodic arrangement of copies of the compact set translated to the lattices points will also be four-fold symmetric, as will be the corresponding Voronoi diagram. Similarly, Voronoi diagrams induced by six-fold symmetric distances over honeycomb lattices will be six-fold symmetric (Figure 13, bottom right).

Let us now assume that $\mathcal{S}$ is three-fold rotationally symmetric, and that we are working with a hexagonal arrangement of sets $\left\{\mathcal{S}_{0}, \ldots, \mathcal{S}_{5}\right\}$. Then, $\mathcal{S}_{i+2}=R_{\frac{2 \pi}{3}} \mathcal{S}_{i}, 0 \leq i \leq 3$, so that the arrangement of these sets is three-fold symmetric. Since a honeycomb lattice is also three-fold symmetric, the Voronoi diagram induced by the star-shape distance associated to $\mathcal{S}$ over such a lattice will also be three-fold symmetric. Interestingly, using a different three-fold symmetric set for for $\mathcal{S}_{1}, \mathcal{S}_{3}, \mathcal{S}_{5}$ preserves this property. This can be leveraged to generate three-fold symmetric hexagonal Voronoi diagrams by interleaving two different three-fold symmetric starshaped sets on a honeycomb lattice.

Noting that a triangular lattice can be obtained by adding a point at the center of each hexagon of a honeycomb lattice, symmetries of Voronoi diagrams over triangular lattices can be directly derived from the honeycomb case. Indeed, if $\mathcal{S}$ is $n$-fold symmetric, with $n=$ 3 or $n=6$, adding $\mathcal{S}$ to the center of a hexagonal arrangement of sets will preserve its $n$-fold symmetry. Therefore, rotational symmetries 

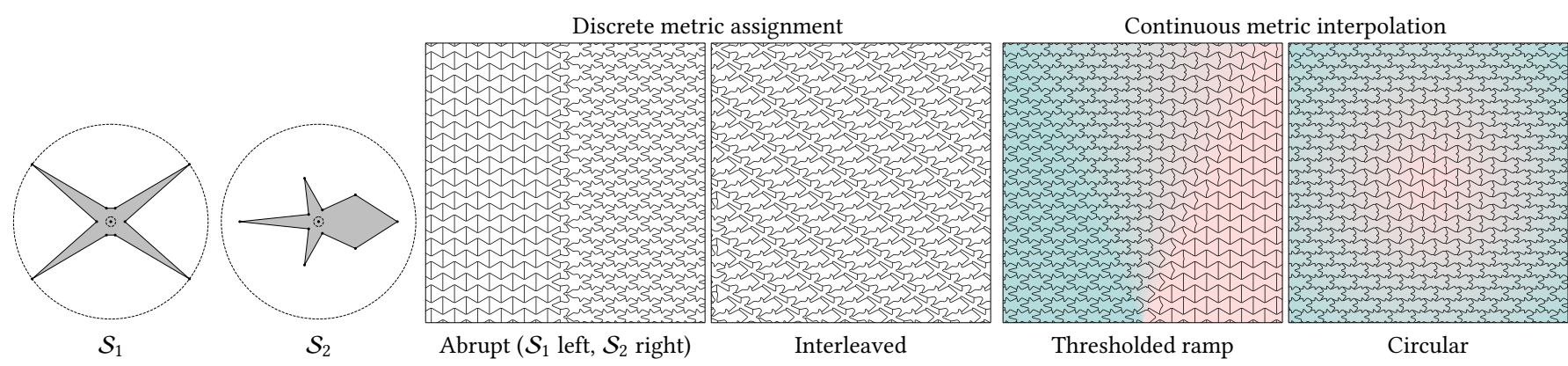

Fig. 10. Networks induced by spatially-varying metrics based on two star-shaped sets $\mathcal{S}_{1}$ and $\mathcal{S}_{2}$. The sites are the grid points of a diagonal square lattice. For the networks resulting from continuous metric interpolation, the color-coding indicates the interpolation weight between $\mathcal{S}_{1}$ and $\mathcal{S}_{2}$. Metric interpolation is performed by linearly blending between the parameter values of the two metrics.
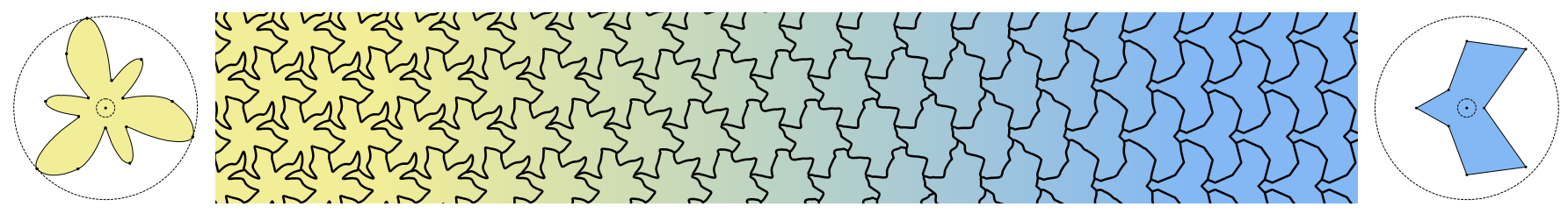

Fig. 11. Continuous interpolation between two star-shaped metrics having different boundary representations (smooth vs. polygonal) and symmetries (three-fold vs. reflection). The sites are the grid points of a triangular lattice.

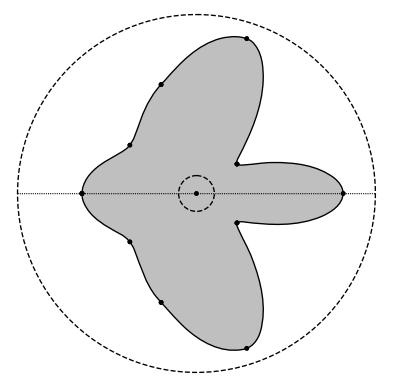

(a) One-axis reflection

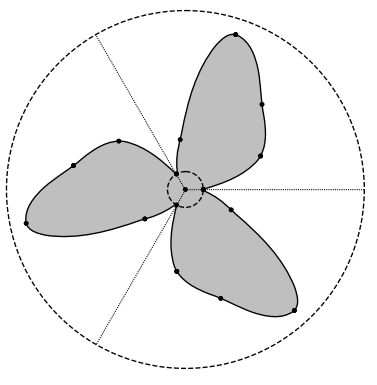

(b) Three-fold rotation

Fig. 12. Distance symmetries.

of Voronoi diagrams over triangular lattices are the same as those of Voronoi diagrams over honeycomb ones.

Reflection symmetry. Let $M_{a}$ denote the reflection across an axis a passing through $O$ and let us assume that $\mathcal{S}$ has mirror, i.e. reflectional, symmetry. Reflecting the translated set $\mathcal{S}_{p}$ is equivalent to translating the set $\mathcal{S}$ to the reflected point $M_{a}(p)$ (see Figure 13, top right). Following the same line of reasoning as above, we can show that the polygonal fan of translated sets $\mathcal{S}_{i}$ exhibits reflectional symmetry with respect to $a$, if $a$ is a reflection axis for the polygon. Thus, the reflectional symmetries shared by the star-shaped metric and the lattice will also be shared by the induced Voronoi diagram.

\section{RESULTS}

We used our method to generate a variety of tilings, both homogeneous and graded, and analyze their elastic response. We first

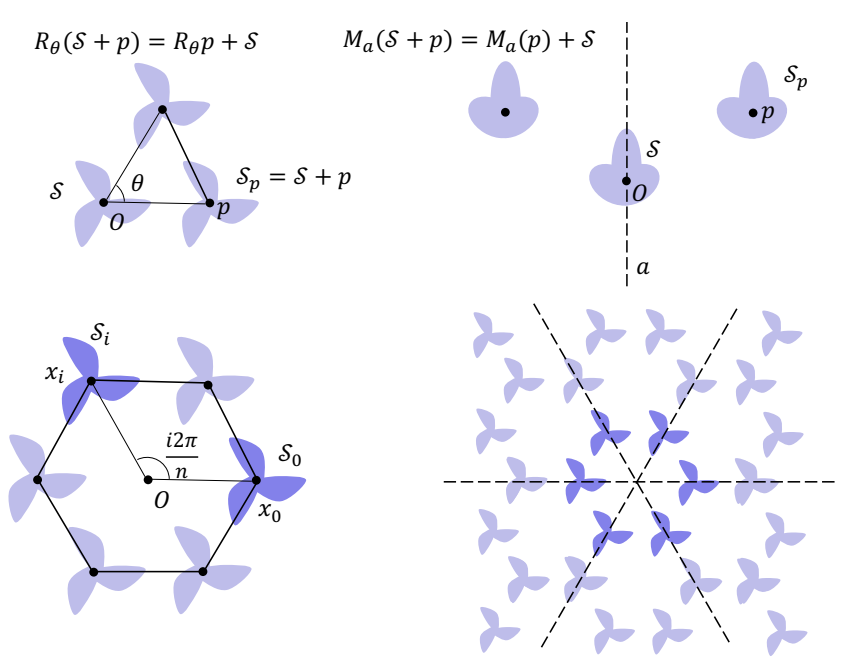

Fig. 13. If a compact set $\mathcal{S}$ is invariant when rotated by $\theta$, then translating the set to $p$ and rotating it by $\theta$ is equivalent to translating the set to the rotated position $R_{\theta} p$ (top, left). If a set is reflection-symmetric with respect to an axis $a$, then the reflected translated set is the set translated to the reflected position $M_{a}(\mathrm{p})$ (top, right). This allows us to deduce symmetry properties of polygonal arrangement of sets (bottom, left) and, by extension, to periodic arrangements of sets (bottom, right).

characterize some sub-spaces of achievable material tensors (Section 4.1). We then turn to graded structures and analyze their elastic properties (Section 4.2). Finally, we present experimental measurements performed on manufactured samples in Section 4.3. 


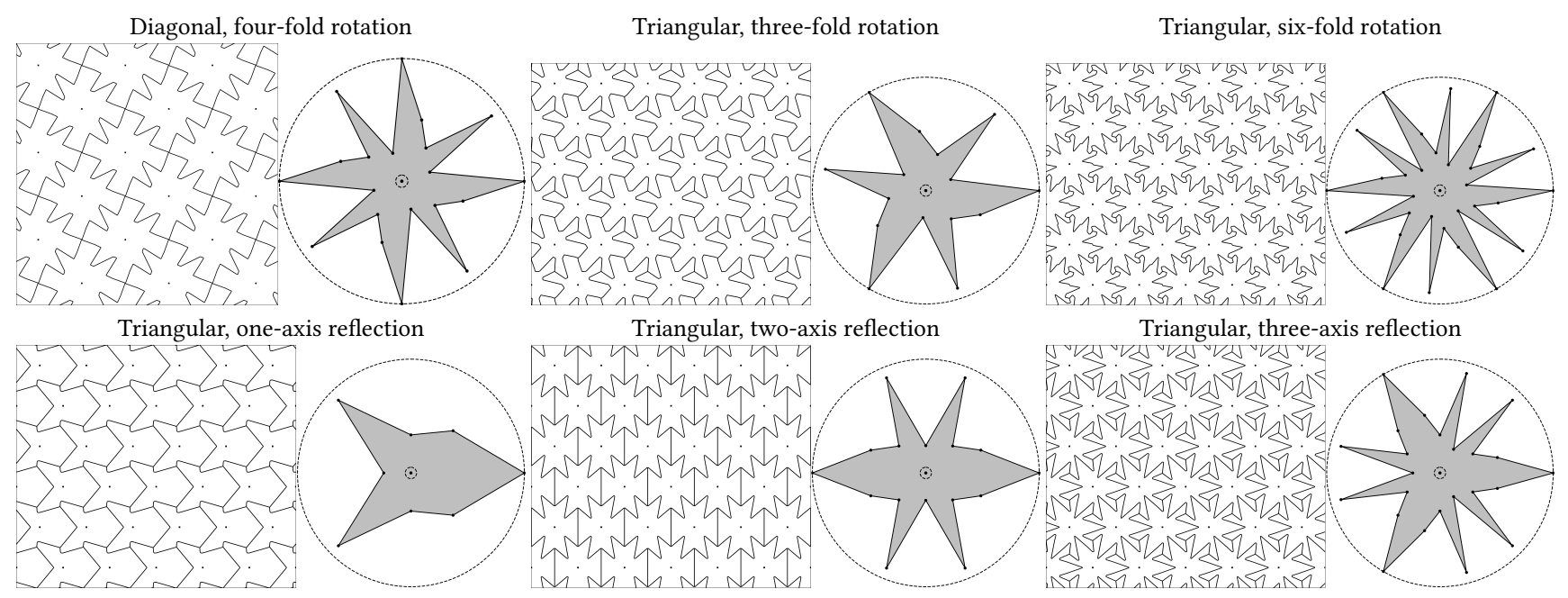

Fig. 14. Representative examples for the different symmetry relations between lattice, metric, and resulting networks given in Table 1.

\begin{tabular}{|c|c|c|}
\hline Lattice type & Metric symmetry & Network symmetry \\
\hline Square & Four-fold rotation & Four-fold rotation \\
Honeycomb/Triangular & Six-fold rotation & Six-fold rotation \\
Honeycomb/Triangular & Three-fold rotation & Three-fold rotation \\
Square/Honeycomb/Triangular & One-axis reflection & One-axis reflection \\
Square/Honeycomb/Triangular & Two-axis reflection & Two-axis reflection \\
Honeycomb/Triangular & Three-axis reflection & Three-axis reflection \\
Honeycomb/Triangular & Six-axis reflection & Six-axis reflection \\
\hline
\end{tabular}

Table 1. Lattice and metric symmetries are reflected in the induced Voronoi networks. See Figure 14 for visual evidence of some of them.

\subsection{Material space}

By combining different metrics and lattice types, our method is able to generate a vast space of Voronoi networks. Here we explore the subspace of materials obtained by combining three typical 2D lattices-diagonal square, triangular, and honeycomb-and metrics having one-axis reflectional and three-fold rotational symmetry. As explained in Section 3.5, for a given lattice there exists a connection between the symmetry of the metric and the symmetry of the obtained Voronoi network. Thus, by pairing metrics with specific symmetries to a given lattice we obtain symmetric networks. This helps us anticipate the resulting elastic properties.

As explained in Section 3.5, symmetric networks can be obtained by proper pairing of metric and lattice. Symmetries in the network, in turn, allow us to categorize the elastic properties of the material. For example, it is well-known that one-axis reflectional symmetry in networks leads to orthotropic materials [Schumacher et al. 2018] while three-fold rotational symmetry yields isotropic materials [Forte and Vianello 1996].

For each choice of lattice and star-shaped distance we run a numerical analysis of the resulting Voronoi network. We perform numerical homogenization in a $2 \mathrm{D}$ rectangular periodic domain. Figure 15 shows the domain adopted for the three tested lattices. We use the publicly available homogenization code of Andreassen and
Andreassen [2014], that computes the homogenized linear elasticity tensor of a 2D image representing a two-phase periodic material. We consider a solid phase with Young's modulus $E=1 P a$ and Poisson's ratio $v=0.3$. Elements corresponding to the void phase are ignored (see [Andreassen and Andreasen 2014, Section 3.1]).
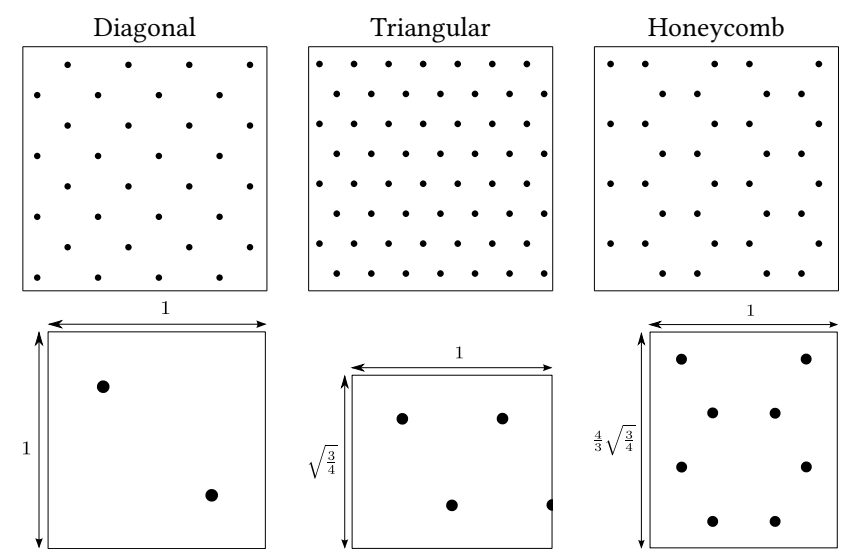

Fig. 15. The three different lattice types used in this work (top) and their corresponding periodic domains (bottom).

We exhaustively sample symmetric distance functions parameterized by 7 equally spaced angles with 3 different radial spans $[0.2,0.6,1.0]$, which leads to $3^{7}=2187$ different metrics. Combined with the diagonal square, triangular and honeycomb lattices as well as polygonal and smooth boundaries, this results in ten databases. We invite the reader to download the databases from the supplemental material data. It contains static html with embedded databases for browsing; a screen shot is shown in Figure 16. Figures 17, 18, and 19 provide some illustrative samples from the triangular and honeycomb lattice databases. 

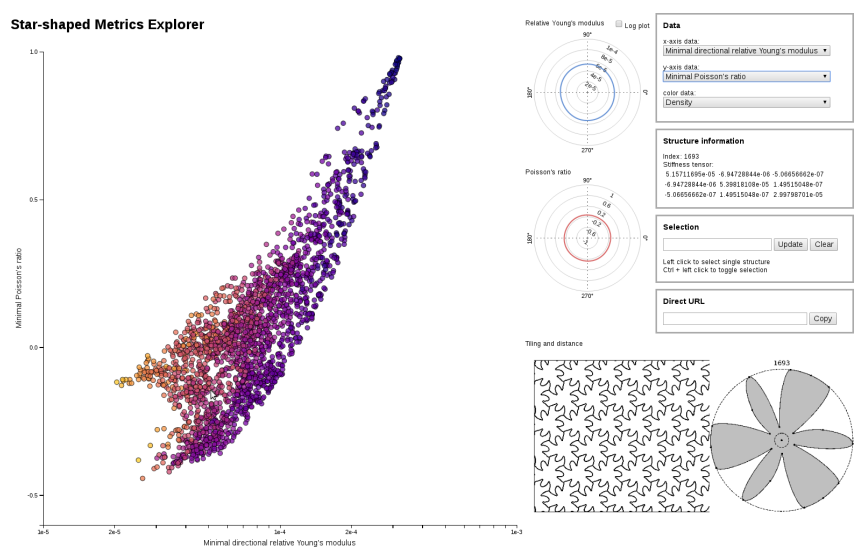

Fig. 16. Interactive visualization of the material space databases. When selecting a sample (colored dots), the corresponding tiling and star-shaped metric appear (right, bottom) alongside two plots its directional Young's modulus and Poisson's ratio.

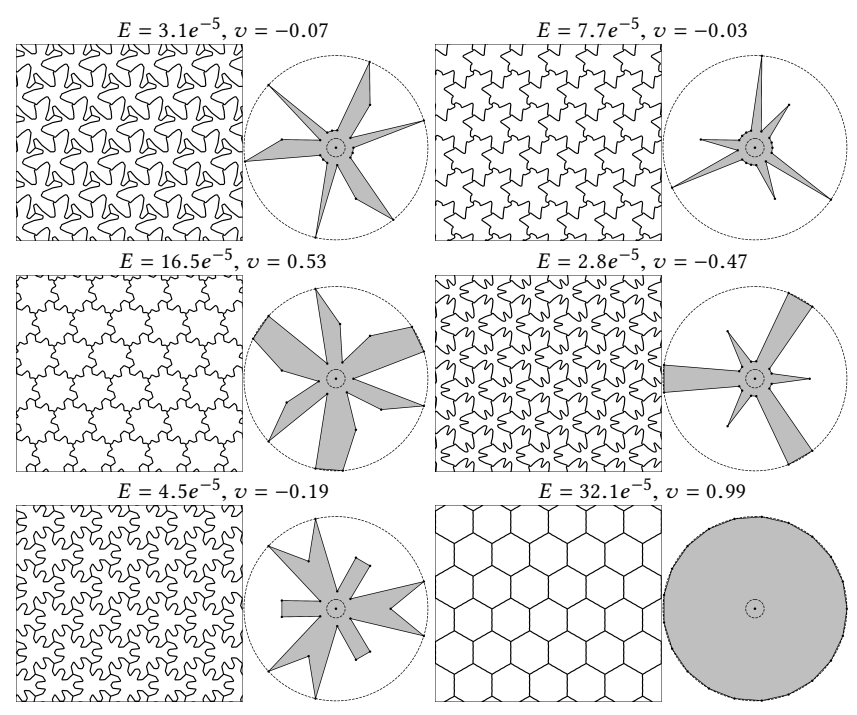

Fig. 17. Isotropic materials obtained by combining triangular lattices with polygonal metrics having three-fold rotational symmetry. For these examples, the Poisson's ratio ranges between -0.47 and 0.99 .

Since we know what material properties to expect from symmetric networks, we can check the databases for any outliers. We determine the symmetry class of the homogenized elasticity tensor (isotropic, tetragonal, orthotropic, anisotropic) numerically using the criteria proposed by de Saxcé and Vallée [2013], using a relative tolerance threshold of $3 \cdot 10^{-6}$. All the databases with one-axis reflection symmetry exhibited an orthotropic tensor as expected. However, the databases with three-fold symmetry contained a few cases that did not exhibit the expected isotropic tensor; $0.8 \%$ for the triangular lattice and $4.4 \%$ for the honeycomb lattice. These outliers can be explained by inaccuracies due to the discrete nature of our growth-based algorithm (see Algorithm 1). Discretization artifacts

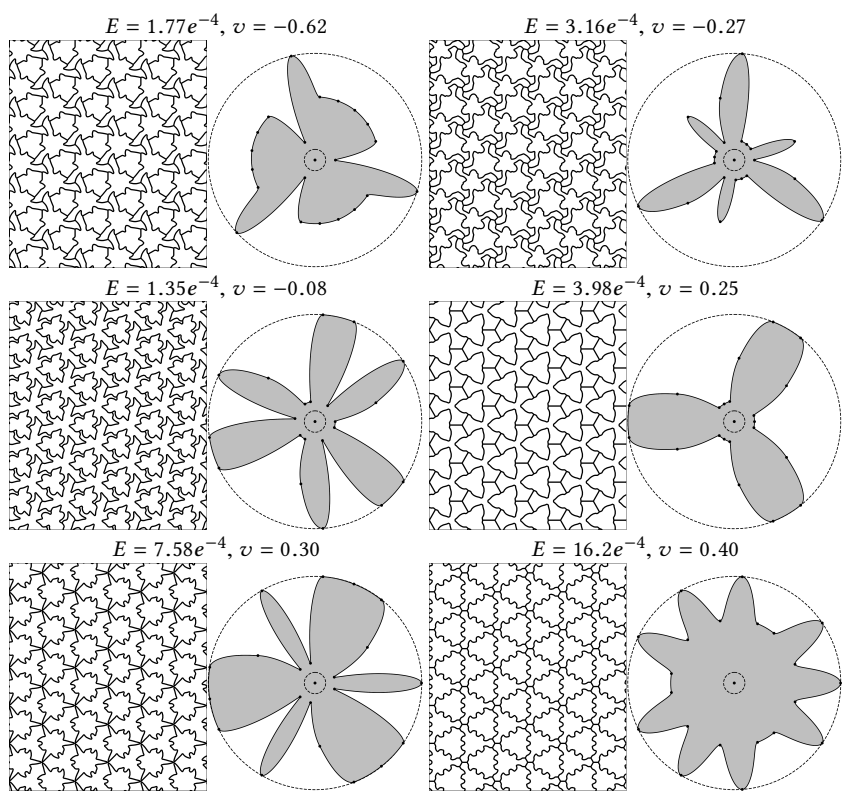

Fig. 18. Isotropic materials obtained by combining honeycomb lattices and smooth metrics with three-fold rotational symmetry. For these examples, the Poisson's ratio ranges between -0.62 and 0.4 .

due to finite grid resolution lead to imperfections in the three-fold symmetry that are reflected in the homogenized tensor. As indicated in Figure 20, such misclassifications can often be resolved by increasing the grid resolution.

\subsection{Interpolation}

Having investigated the material spaces induced by lattice and metric symmetries, we now proceed to interpolating between different metrics as described in Section 3.4.

The central question in this context is how the geometry of the Voronoi network and its elastic properties evolve during interpolation. Figure 21 illustrates the linear interpolation between two distance functions with tilings for different interpolation weights. It is worth noting that, while material constants change in a continuous but non-monotonic manner, the material tensor remains orthotropic as expected. Thus, smooth interpolation in metric space does not imply equally smooth changes in geometry. Figure 22 shows an example for which the elastic properties change abruptly, triggered by an abrupt change of geometry in the network.

The above example illustrates that linear interpolation in metric space does not, in general, translate into controlled monotonic interpolation of elastic properties. Nevertheless, this approach still performs well as a tool for designing new patterns and exploring their macro-mechanical properties.

\subsection{D-Printed samples}

To verify that the generated networks behave as predicted by numerical homogenization, we printed a selection of sample structures and tested their macro-mechanical properties. For generality with 

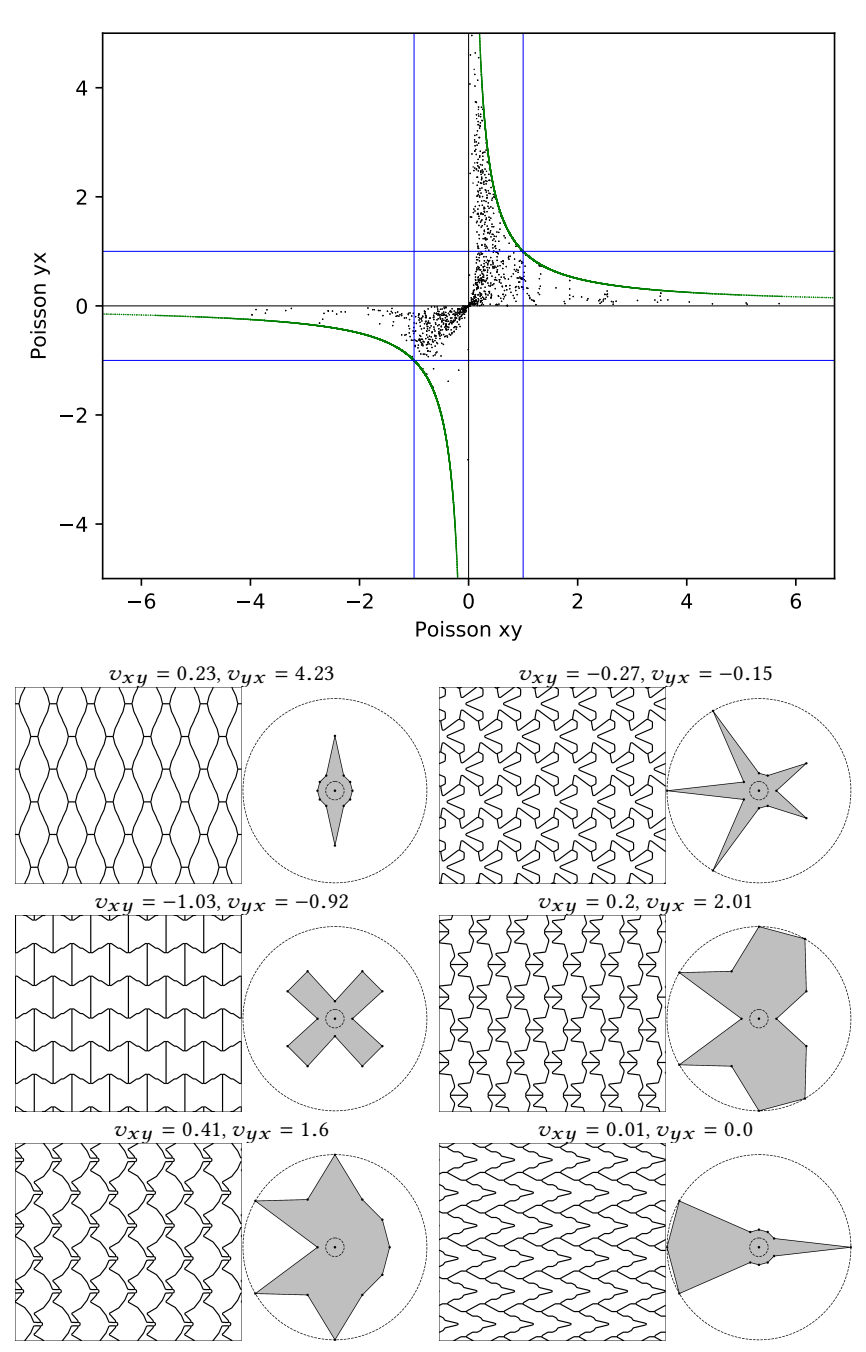

Fig. 19. Orthotropic materials obtained when combining triangular lattices with metrics having polygonal boundaries and one-axis reflectional symmetry. Top plot: Poisson's ratio coverage with theoretical bounds $\frac{E_{x}}{E_{y}}>v_{x y}^{2}, \frac{E_{y}}{E_{x}}>v_{y x}^{2}$ shown in green (see [Ting and Chen 2005]). One of the materials that emerges from this space is a well known re-entrant structure (second row, left).

respect to the filament used, we only report relative stiffness values, i.e., the ratio between the stiffness of a given sample and the average across all tested samples. The results of these tests are shown in Figure 23. It can be seen that prediction and experiments produce the same ordering with ratios agreeing closely, except for Sample 4. The larger discrepancy for this structure is likely due to the fact that it is much softer than the other ones, which increases the relative impact of inaccuracies during printing and testing. In order to remain within the linear-elastic regime of the networks, we used small strains of $3 \%$ for testing. However, to better illustrate the effect of Poisson's ratio, we show images for larger strains in Figure 23.

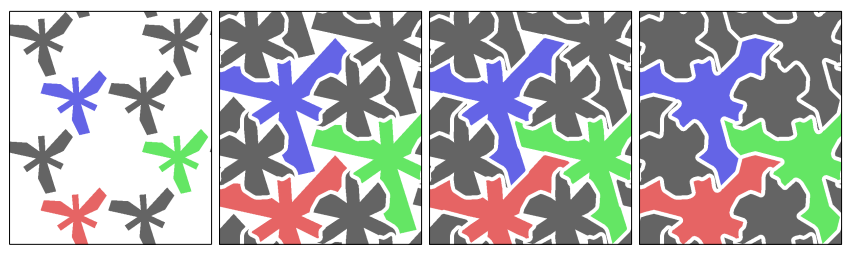

$\times 2$ resolution grid

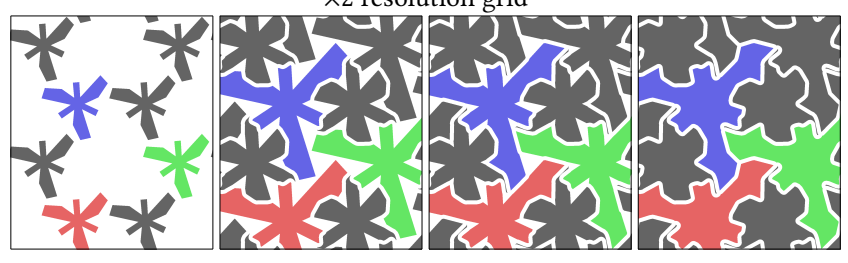

Fig. 20. Top: an outlier in the database exhibiting a non-isotropic material tensor. This network was generated using a honeycomb lattice paired with a three-fold symmetric distance. As can be seen from the evolution of the Voronoi growth process (from left to right), the coarse discretization leads to one cell pair (red-blue) merging in a different way than the two other ones (blue-green, green-red). This break of symmetry in the network translates into a non-isotropic material tensor. Bottom: increasing the grid resolution resolves this problem.

Beyond pure validation, we also printed a number sample networks that illustrate interesting effects obtained by combining various properties. Figure 24 shows a homogeneous network, obtained using a honeycomb lattice and a three-fold symmetric distance, that exhibits a negative Poisson's ratio. The example shown in Figure 25 is a heterogeneous structure created using discrete metric assignment. It features a central band with negative Poisson's ratio surrounded by two bands with positive Poisson's ratio. Figure 26 shows a heterogeneous network created with continuous metric interpolation. This structure consists of two orthotropic regions: a disc a its center with high/low tensile stiffness in the vertical/horizontal direction, and a surrounding region whose material exhibits roughly the opposite alignment of principal tensile stiffnesses.

\section{LIMITATIONS, FUTURE WORK}

We presented a method for designing mechanical metamaterials based on the novel concept of Voronoi diagrams induced by starshaped metrics. The inherent support for interpolation gives rise to a large, continuous space of patterns and it opens the door to smoothly graded structures. While our results indicate the potential for metamaterial design, there are a number of limitations and open questions that merit further investigation.

Due to the coupled relation between distance and lattice symmetry (see Section 3.5), the principal directions of an orthotropic material cannot be chosen independently from those of the lattice. As illustrated in Figure 27, breaking the alignment between lattice and metric symmetries will generally lead to anisotropic material behavior [Schumacher et al. 2018].

Our growth algorithm guarantees that individual Voronoi cells are always connected. However, this is not sufficient for guaranteeing connectedness of the resulting network. While we conjecture that disconnected networks cannot occur when using the same metric 

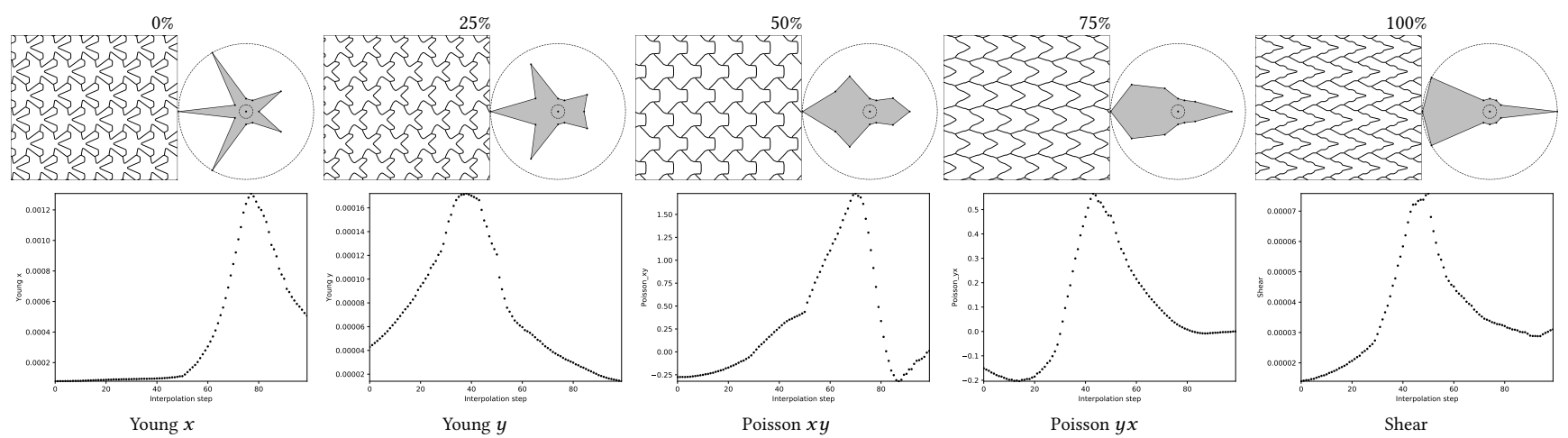

Fig. 21. Top: Linear interpolation between two metrics (with weights as indicated relative to second metric) using a triangular lattice and distance functions with one-axis reflectional symmetry (orthotropic case). See accompanying video for a more detailed visualization of the interpolation. Bottom: In this example, the material properties change continuously but in a non-monotonic fashion.
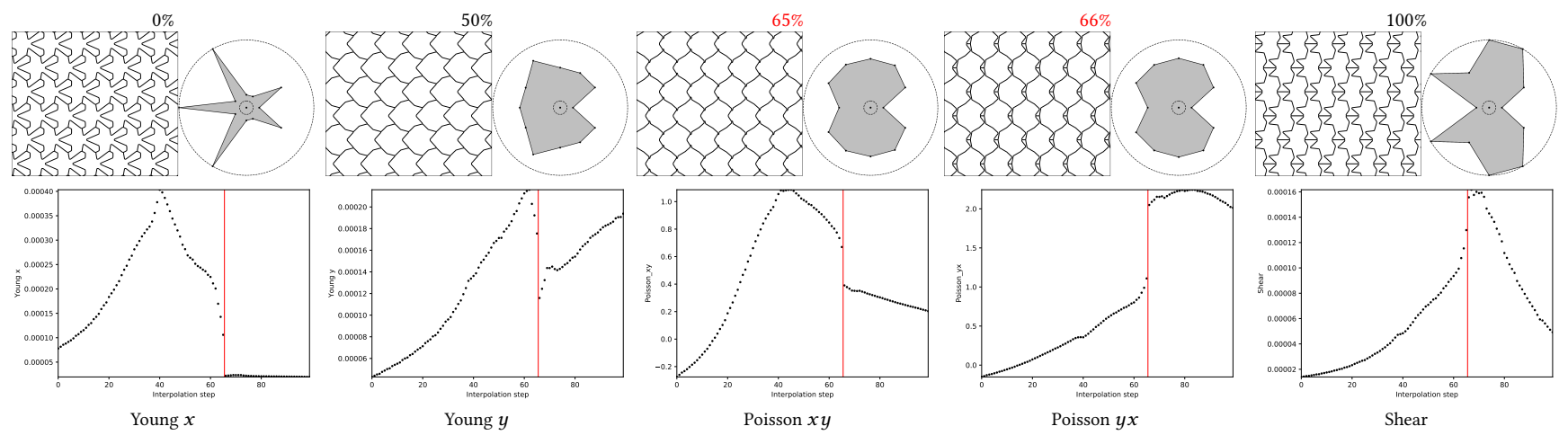

Fig. 22. Same settings as in Figure 21, but with a different second distance function (far right). There is a rapid change in material properties (red line between $65 \%$ to $66 \%$ ) due to a sudden change in shape of the Voronoi cells. See also accompanying video for a more detailed visualization of this interpolation process.

throughout the domain, it is easy to construct counterexamples in the general case: when using spatially varying metrics, the metric of a given site can be scaled up such that its Voronoi cell completely engulfs the cell of a neighboring site. Although we have never observed this problem in our experiments, the growth algorithm could be modified to track connectivity and flag problems. As future work, it would be interesting to study which guarantees can be obtained regarding isolated Voronoi cells as a function of the metric.

Regarding the elastic behavior of our Voronoi networks, a broader exploration of the material space [Zhu et al. 2017] and extensions into the nonlinear regime [Schumacher et al. 2018] will increase the understanding of the space of material properties that can be achieved. However, it is evident that the space of pattern geometries spanned by star-shaped Voronoi diagrams does not encompass all tileable geometries and elastic behaviors. For instance, it does not cover the spirals patterns of Malomo et al. [2018] which achieve very low stiffness.

While we have performed initial experiments with spatially varying metrics, we have not attempted to systematically analyze the material properties of the corresponding graded structures. Possible investigations in this direction include an analysis of the macromechanical properties for graded structures created using prescribed interpolation (e.g., for aesthetic reasons) and the automated design of networks with the desired grading of mechanical properties. Furthermore, we have so far only considered linear interpolation between star-shaped distances, but more advanced interpolation schemes might offer advantages with respect to smoothness and monotonicity of the homogenized elasticity tensor (see Figure 22).

Finally, our formulation naturally extends to 3D network materials, requiring only straight-forward generalizations of star-shaped metrics and the growth algorithm. The increase in dimension also entails a larger number of possible combinations between metric and lattice symmetries, which seems worthwhile exploration.

\section{ACKNOWLEDGMENTS}

This work was partly supported by ANR ${ }^{1}$ MuFFin (ANR-17-CE100002), the French PIA project "Lorraine Universite d'Excellence" (ANR-15-IDEX-04-LUE), the Natural Sciences and Engineering Research Council of Canada (NSERC), and the Fonds de recherche du Québec nature et technologie. We thank Noémie Vennin for help with video editing.

\footnotetext{
${ }^{1}$ Agence nationale de la recherche
} 
Specimen 1

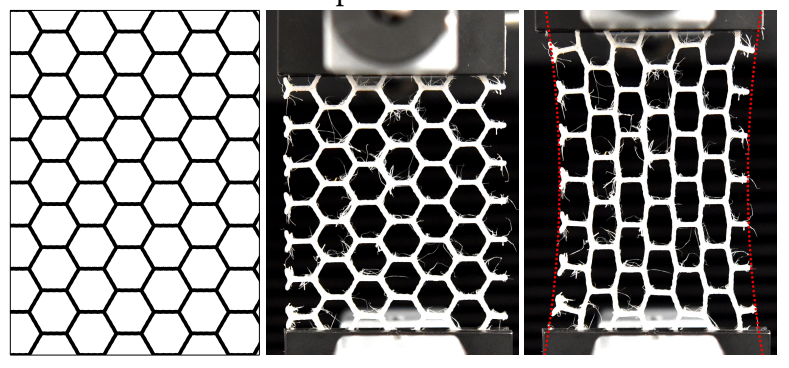

Specimen 2

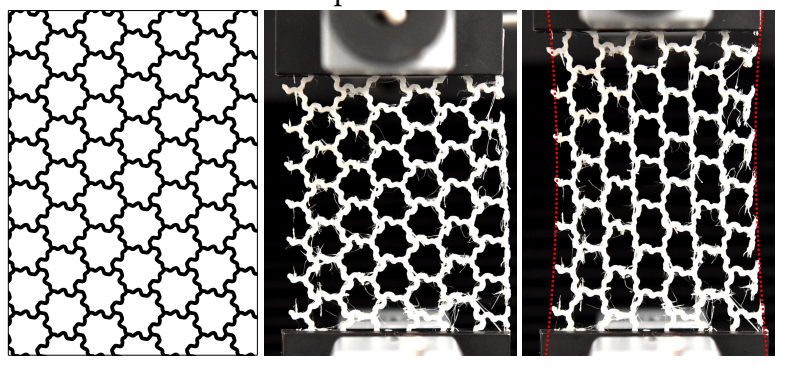

Specimen 3

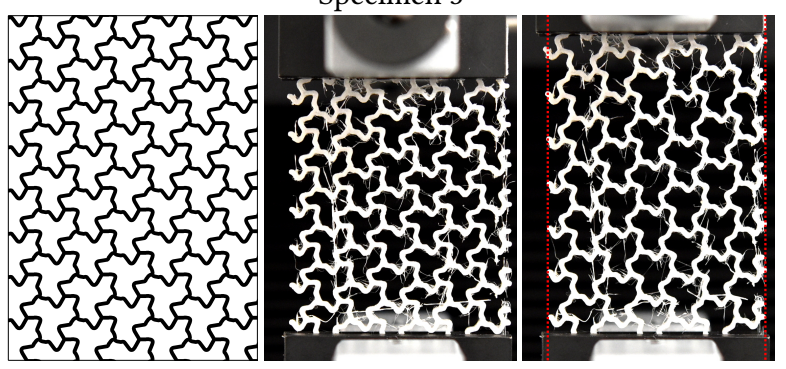

Specimen 4

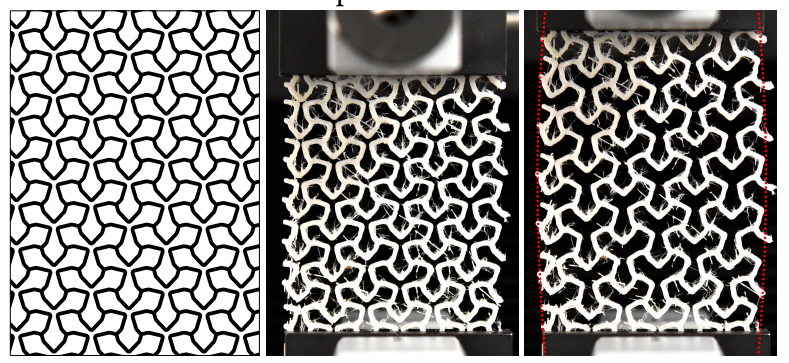

\begin{tabular}{c|cc|ccc|} 
& \multicolumn{2}{|c|}{ Experimental } & \multicolumn{4}{c|}{ Simulation } \\
$\mathrm{Id}$ & $E(\mathrm{MPa})$ & Ratio $E$ & $E$ & Ratio $E$ & $v$ \\
\hline 1 & 0.32 & 1.80 & 0.00764 & 2.03 & 0.91 \\
2 & 0.21 & 1.18 & 0.00465 & 1.23 & 0.57 \\
3 & 0.11 & 0.62 & 0.00203 & 0.54 & -0.07 \\
4 & 0.07 & 0.39 & 0.00070 & 0.18 & -0.42
\end{tabular}

Fig. 23. Tensile testing with an Instron 3345 . The four specimens with dimensions $69.8 \times 50 \times 3 \mathrm{~mm}$ where printed with an Ultimaker 3 using the NinjaFlex 85A filament (thermoplastic polyurethane). The first and third column of the table show the experimental (linear regime) and the numerical (linear homogenization) Young's moduli. The second and the fourth column show the experimental and numerical relative ratio, obtained by diving the corresponding Young modulus with the average of the four specimens. The photos show the resting position (middle) and a tensioned one with high elongation of $10 \mathrm{~mm}$, that visually reveal (dotted red line) the coherency of the simulated Poisson's ratio with the experimental.

ACM Trans. Graph., Vol. 38, No. 4, Article 82. Publication date: July 2019.
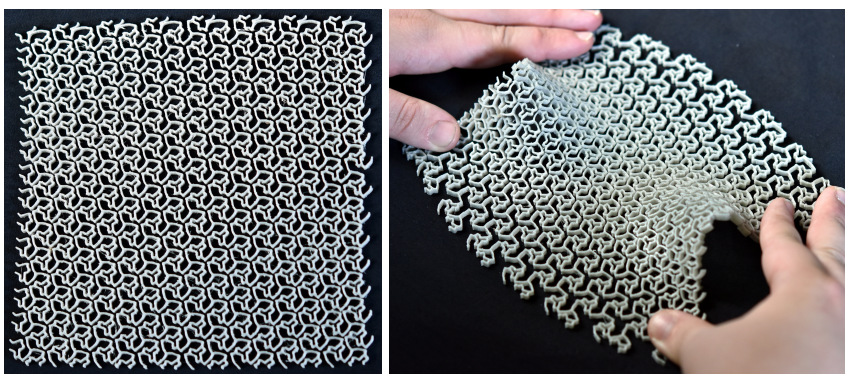

Fig. 24. A network material with negative Poisson's ratio.
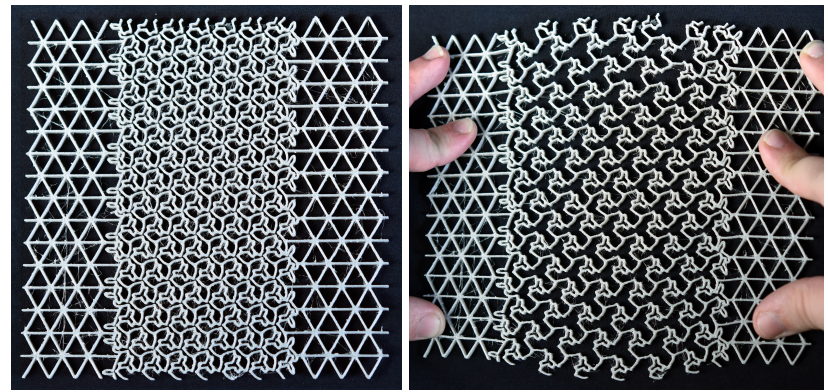

Fig. 25. A heterogeneous sheet with one central band having negative Poisson's ratio.
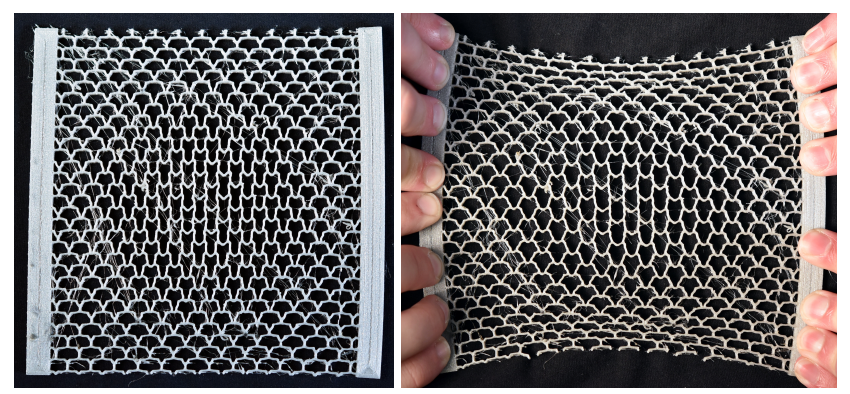

Fig. 26. A heterogeneous network with an interior region that exhibits lower Young's modulus in the horizontal direction than the surrounding region.

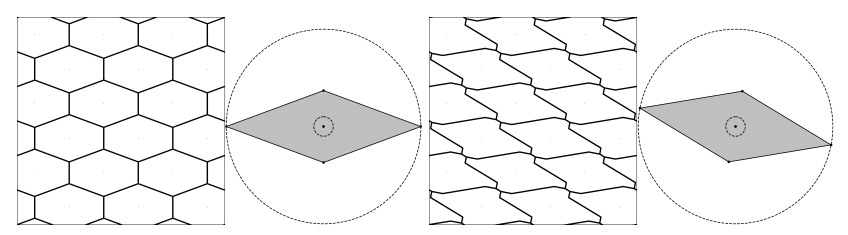

Fig. 27. Diagonal square lattice paired with a metric with one-axis reflectional symmetry. Left: the symmetry axes of metric and lattice are aligned, resulting in an orthotropic material. Right: a slight rotation of the metric reduces the symmetry of the network to two-fold rotational and, consequently, leads to anisotropic behavior.

\section{REFERENCES}

Erik Andreassen and Casper Schousboe Andreasen. 2014. How to Determine Composite Material Properties Using Numerical Homogenization. Computational Materials 
Science 83 (2014), 488-495.

Erik Andreassen, Boyan S. Lazarov, and Ole Sigmund. 2014. Design of manufacturable 3D extremal elastic microstructure. Mechanics of Materials 69, 1 (2014), 1-10.

Melvin Avrami. 1939. Kinetics of phase change. I General theory. The fournal of chemical physics 7, 12 (1939), 1103-1112.

Sahab Babaee, Jongmin Shim, James C. Weaver, Elizabeth R. Chen, Nikita Patel, and Katia Bertoldi. 2013. 3D Soft Metamaterials with Negative Poisson's Ratio. Advanced Materials 25, 36 (2013), 5044-5049.

Katia Bertoldi, Vincenzo Vitelli, Johan Christensen, and Martin van Hecke. 2017. Flexible mechanical metamaterials. Nature Reviews 2 (2017), 17066.

Bernd Bickel, Moritz Bächer, Miguel A. Otaduy, Hyunho Richard Lee, Hanspeter Pfister, Markus Gross, and Wojciech Matusik. 2010. Design and Fabrication of Materials with Desired Deformation Behavior. ACM Trans. Graph. (Proc. SIGGRAPH) 29, 4 Article 63 (2010).

J. Blömer and K. Kohn. 2018. Voronoi Cells of Lattices with Respect to Arbitrary Norms. SIAM Journal on Applied Algebra and Geometry 2, 2 (2018), 314-338.

Weikai Chen, Xialong Zhang, Shiqing Xin, Yang Xia, Sylvain Lefebvre, and Wenping Wang. 2016. Synthesis of Filigrees for Digital Fabrication. ACM Trans. Graph. (Proc. SIGGRAPH) 35, 4 (2016).

A.G. Corbalan, M. Mazon, and T. Recio. 1996. Geometry of bisectors for strictly convex distances. Int. 7. Comput. Geom. Appl. 06, 01 (1996), 45-58.

Corentin Coulais, Eial Teomy, Koen de Reus, Yair Shokef, and Martin van Hecke. 2016. Combinatorial design of textured mechanical metamaterials. Nature 535 (2016) 529-532.

Andrew D. Cramer, Vivien J. Challis, and Anthony P. Roberts. 2016. Microstructure interpolation for macroscopic design. Struct. Multidiscip. Opt. 53, 3 (2016), 489-500.

Géry de Saxcé and Claude Vallée. 2013. Invariant Measures of the Lack of Symmetry with Respect to the Symmetry Groups of 2D Elasticity Tensors. Fournal of Elasticity 111, 1 (2013), 21-39.

Michel Deza and Mathieu Dutour Sikirić. 2015. Voronoi polytopes for polyhedral norms on lattices. Discrete Applied Mathematics 197 (2015), 42 - 52.

Michel Marie Deza and Elena Deza. 2013. Voronoi Diagram Distances. Springer Berlin Heidelberg, Berlin, Heidelberg, 339-347.

Zongliang Du, Xiao-Yi Zhou, Renato Picelli, and H Alicia Kim. 2018. Connecting Microstructures for Multiscale Topology Optimization With Connectivity Index Constraints. Fournal of Mechanical Design 140, 11 (2018), 111417.

Jérémie Dumas, An Lu, Sylvain Lefebvre, Jun Wu, and Christian Dick. 2015. By-example Synthesis of Structurally Sound Patterns. ACM Trans. Graph. (Proc. SIGGRAPH) 34 4, Article 137 (2015)

Herbert Edelsbrunner and Ernst Peter Mücke. 1990. Simulation of simplicity: a technique to cope with degenerate cases in geometric algorithms. ACM Trans. Graph. 9, 1 (1990), 66-104.

Sandra Forte and Maurizio Vianello. 1996. Symmetry classes for elasticity tensors. fournal of Elasticity 43, 2 (01 May 1996), 81-108.

F. Fritsch and R. Carlson. 1980. Monotone Piecewise Cubic Interpolation. SIAM 7 Numer. Anal. 17, 2 (1980), 238-246.

Eric Garner, Helena Kolken, Charlie Wang, Amir Zadpoor, and Jun Wu. 2019. Compatibility in Microstructural Optimization for Additive Manufacturing. Additive Manufacturing 26 (2019), 65-75.

J. N. Grima and K. E. Evans. 2000. Auxetic behavior from rotating squares. Fournal of Materials Science Letters 19, 17 (2000), 1563-1565.

Peter Gruber. 1974. Über kennzeichnende Eigenschaften von euklidischen Räumen und Ellipsoiden. I. fournal für die reine und Angewandte Mathematik 265 (1974), 61-83.

Branko Grünbaum and G C Shephard. 1986. Tilings and Patterns. W. H. Freeman \& Co., New York, NY, USA

Behrooz Hassani and Ernest Hinton. 1998. A review of homogenization and topology optimization I-homogenization theory for media with periodic structure. Computers \& Structures 69, 6 (1998), 707-717.

C. He, H. Martini, and S. Wu. 2011. On Bisectors for Convex Distance Functions. In Eight International Symposium on Voronoi Diagrams in Science and Engineering. 23-30.

Christian Icking, Rolf Klein, Ngoc-Minh Lé, and Lihong Ma. 1995. Convex distance functions in 3-space are different. Fundamenta Informaticae 22, 4 (1995), 331-352.

Christian Icking, Rolf Klein, Lihong Ma, Stefan Nickel, and Ansgar Weißler. 2001. On bisectors for different distance functions. Discrete Appl. Math. 109, 1 (2001), 139-161.

Alexandra Ion, Johannes Frohnhofen, Ludwig Wall, Robert Kovacs, Mirela Alistar, Jack Lindsay, Pedro Lopes, Hsiang-Ting Chen, and Patrick Baudisch. 2016. Metamaterial Mechanisms. In Proceedings of the 29th Annual Symposium on User Interface Software and Technology (UIST '16). 529-539.

Alexandra Ion, Robert Kovacs, Oliver S. Schneider, Pedro Lopes, and Patrick Baudisch 2018. Metamaterial Textures. In Proceedings of the 2018 CHI Conference on Human Factors in Computing Systems. Article 336, 336:1-336:12 pages.

Craig S. Kaplan and David H. Salesin. 2000. Escherization. In Proceedings of the 27th Annual Conference on Computer Graphics and Interactive Techniques (SIGGRAPH '00). 499-510.

H MA Kolken and AA Zadpoor. 2017. Auxetic mechanical metamaterials. RSC Advances 7, 9 (2017), 5111-5129.
Mina Konaković, Keenan Crane, Bailin Deng, Sofien Bouaziz, Daniel Piker, and Mark Pauly. 2016. Beyond Developable: Computational Design and Fabrication with Auxetic Materials. ACM Trans. Graph. (Proc. SIGGRAPH) 35, 4 (2016).

Mina Konaković-Luković, Julian Panetta, Keenan Crane, and Mark Pauly. 2018. Rapid Deployment of Curved Surfaces via Programmable Auxetics. ACM Trans. Graph. 37, 4, Article 106 (2018).

Roderic Lakes. 1987. Foam Structures with a Negative Poisson's Ratio. Science 235, 4792 (1987).

Jianxing Liu and Yihui Zhang. 2018. Soft network materials with isotropic negative Poisson's ratios over large strains. Soft Matter 14 (2018), 693-703. Issue 5.

Lihong Ma. 2000. Bisectors and Voronoi diagrams for convex distance functions. Ph.D. Dissertation. Fernuniversität, Fachbereich Informatik.

Luigi Malomo, Jesús Pérez, Emmanuel Iarussi, Nico Pietroni, Eder Miguel, Paolo Cignoni, and Bernd Bickel. 2018. FlexMaps: Computational Design of Flat Flexible Shells for Shaping 3D Objects. ACM Trans. Graph. (Proc. SIGGRAPH Asia) 37, 6, Article 241 (2018), 241:1-241:14 pages.

Jonàs Martínez, Jérémie Dumas, and Sylvain Lefebvre. 2016. Procedural Voronoi Foams for Additive Manufacturing. ACM Trans. Graph. (Proc. SIGGRAPH) 35, 4 (2016).

Jonàs Martínez, Jérémie Dumas, Sylvain Lefebvre, and Li-Yi Wei. 2015. Structure and Appearance Optimization for Controllable Shape Design. ACM Trans. Graph. (Proc. SIGGRAPH Asia) 34, 6 (2015).

Jonàs Martínez, Samuel Hornus, Haichuan Song, and Sylvain Lefebvre. 2018. Polyhedral Voronoi Diagrams for Additive Manufacturing. ACM Trans. Graph. (Proc. SIGGRAPH) 37, 4, Article 129 (2018).

Jonàs Martínez, Haichuan Song, Jérémie Dumas, and Sylvain Lefebvre. 2017. Orthotropic $k$-nearest Foams for Additive Manufacturing. ACM Trans. Graph. (Proc. SIGGRAPH) 36, 4, Article 121 (2017)

H. Martini and K.J. Swanepoel. 2004. The geometry of Minkowski spaces: A survey. Part II. Expositiones Mathematicae 22, 2 (2004), 93 - 144

Luke Mizzi, E.M. Mahdi, Kirill Titov, Ruben Gatt, Daphne Attard, Kenneth E. Evans, Joseph N. Grima, and Jin-Chong Tan. 2018. Mechanical metamaterials with starshaped pores exhibiting negative and zero Poisson's ratio. Materials \& Design 146 (2018), 28-37.

Julian Panetta, Abtin Rahimian, and Denis Zorin. 2017. Worst-case Stress Relief for Microstructures. ACM Trans. Graph. (Proc. SIGGRAPH) 36, 4 (2017).

Julian Panetta, Qingnan Zhou, Luigi Malomo, Nico Pietroni, Paolo Cignoni, and Denis Zorin. 2015. Elastic Textures for Additive Fabrication. ACM Trans. Graph. (Proc. SIGGRAPH) 34, 4, Article 135 (2015).

Christian Schumacher, Bernd Bickel, Jan Rys, Steve Marschner, Chiara Daraio, and Markus Gross. 2015. Microstructures to Control Elasticity in 3D Printing. ACM Trans. Graph. (Proc. SIGGRAPH) 34, 4, Article 136 (2015).

Christian Schumacher, Steve Marschner, Markus Cross, and Bernhard Thomaszewski. 2018. Mechanical Characterization of Structured Sheet Materials. ACM Trans. Graph. (Proc. SIGGRAPH) 37, 4, Article 148 (2018).

Christian Schumacher, Bernhard Thomaszewski, and Markus Gross. 2016. Stenciling: Designing Structurally-Sounds Surfaces with Decorative Patterns. Computer Graphics Forum 35, 5 (Aug. 2016), 101-110.

Ole Sigmund. 1997. On the Design of Compliant Mechanisms Using Topology Optimization. Mechanics of Structures and Machines 25, 4 (1997), 493-524.

TCT Ting and Tungyang Chen. 2005. Poisson's ratio for anisotropic elastic materials can have no bounds. Q. J. Mech. Appl. Math 58, 1 (2005), 73-82.

Jussi Väisälä. 2013. Slopes of bisectors in normed planes. Beiträge zur Algebra und Geometrie 54, 1 (2013), 225-235.

F. Wang, O. Sigmund, and J.S. Jensen. 2014. Design of materials with prescribed nonlinear properties. f. Mech. Phys. Solids 69, 4 (2014), 156-174.

Yiqiang Wang, Feifei Chen, and Michael Yu Wang. 2017. Concurrent design with connectable graded microstructures. Comput. Methods Appl. Mech. Eng. 317 (2017), 84-101.

Yiqiang Wang, Lei Zhang, Stephen Daynes, Hongying Zhang, Stefanie Feih, and Michael Yu Wang. 2018. Design of graded lattice structure with optimized mesostructures for additive manufacturing. Materials \& Design 142 (2018), 114 - 123.

Hongyi Xu, Yijing Li, Yong Chen, and Jernej Barbič. 2015. Interactive Material Design Using Model Reduction. ACM Trans. Graph. 34, 2 (2015), 18:1-18:14.

Rhaleb Zayer, Daniel Mlakar, Markus Steinberger, and Hans-Peter Seidel. 2018. Layered Fields for Natural Tessellations on Surfaces. ACM Trans. Graph. (Proc. SIGGRAPH Asia) 37, 6, Article 264 (2018).

Jonas Zehnder, Stelian Coros, and Bernhard Thomaszewski. 2016. Designing Structurally-sound Ornamental Curve Networks. ACM Trans. Graph. (Proc. SIGGRAPH) 35, 4, Article 99 (2016).

Yan Zhang, Hao Li, Mi Xiao, Liang Gao, Sheng Chu, and Jinhao Zhang. 2018. Concurrent topology optimization for cellular structures with nonuniform microstructures based on the Kriging metamodel. Struct. Multidiscip. Opt. (2018).

Shiwei Zhou and Qing Li. 2008. Design of graded two-phase microstructures for tailored elasticity gradients. F. Mater. Sci. 43, 15 (2008), 5157

Bo Zhu, Mélina Skouras, Desai Chen, and Wojciech Matusik. 2017. Two-Scale Topology Optimization with Microstructures. ACM Trans. Graph. 36, 4 (2017). 